\title{
MOVIE OF COMET KOHOUTEK (1973f) AS OBSERVED NEAR MINIMUM ELONGATION BY THE HAO CORONAGRAPH ABOARD SKYLAB
}

\author{
E. Hildner, J. T Gosling, R. M. MacQueen, R. H. Munro, A. I. Poland, and C. L. Ross
}

This paper consisted of a running commentary by Ernest Hildner on the movie as it was shown. The data from the movie has not yet been fully analyzed and will be published elsewhere. Discussion immediately followed the movie. (ed.) 


\section{DISCUSSION}

Z. Sekanina: I just wonder whether there are any specific plans as to the reduction of the film. The reason that $I^{\prime} \mathrm{m}$ asking is that $\mathrm{I}$ am in progress of studying the comet from ground-based data, and I will present some preliminary results from photometric study that I did on Dr. Miller's photographs. I just feel that we have a beautiful opportunity here to tie the observations from Skylab down at the time we couldn't see the comet from the ground, with the ground-based observations at the time when Skylab was working.

E. Hildner: After the first of the year, Dr. Keller is going to work very heavily with us, and I believe that's the subject on which we're going to concentrate. Perhaps you'd like to talk to that?

H. Keller: My comment on this would be that very shortly we should combine ground-based observations with Skylab observations in order to cover a larger heliocentric distance interval, because the anti-tail is clearly to be seen. I think about the 1st of January, - is that right, Ernie? About the 1st of January you can see the anti-tail on the Skylab photographs, and therefore we can go over to ground-based observations, which are beginning just about in the first of January.

I think that has to be done, to combine those.

E. Hildner: One of the interesting things is that the anti-tail is already visible in the Skylab photographs late on the 28th of December, about 10 minutes 'til midnight on the 28th. Within twelve hours of perihelion we have observations of the anti-tail.

M. Dubin: You did indicate that you have polarization data throughout the entire period, and in an earlier paper this morning, described by Jurgen Rahe and Weinberg, they find a polarization change on Mkeya-Seki which is quite substantial. What are your plans on the polarization reductions?

E. Hildner: We had very few plans until I heard that paper this morning, and now I have not formulated any since then. We'll certainly look for the polarization. So far we've concentrated, inasmuch as we've been able to do anything on the straight photometry, unpolarized photometry. I hope to get together with Dr. Weinberg and get a copy of what he said this morning.

We have rather a wide band-pass, and I have the impression that your band-pass is quite narrow, and you saw different polarizations in different colors, different wavelengths, and I'm not sure how that will reflect itself in our observations, which cover about 3400 angstroms. 


\section{DISCUSSION (Continued)}

B. Donn: That could make a problem in interpreting the data, because the polarization is farily wavelength-sensitive, and in a very broad band-you're going to have a problem. You have enough problems in reducing this data, taking a size distribution of the particles into account. If you have to combine that with a large wavelength range, it certainly limits what can be done, but since this is the only data we have near perihelion, it's certainly worthwhile making an effort to see what can come out of it.

E. Hildner: Well, we certainly will look to see what the polarization does and - what the polarization is as a function of time, and as a function of phase angle and whatever. Where we go from there, I think will wait on what those measurements show us.

M. Dubin: Apparently there's a phase change on the polarization of almost 180 degrees near perihelion, for the same particles possibly, in the coma, so one could study a substantial -

Voice: Not in the coma, no.

M. Dubin: Well, it's close to the coma. It's the coma also, you observe, that has a polarization.

J. L. Weinberg: This is far out in the tail. Polarization reversal occurs at 130 degrees scattering angle, nominally, and about 11 degrees from the nucleus, so it's far out. 